\title{
Roadmap to clinical translation: insights from a UK regenerative medicine platform workshop on mesenchymal stromal cells
}

\author{
Curtis O Asante ${ }^{*}, 1$ \& Francesco Dazzi² \\ ${ }^{1}$ Centre for Stem Cells and Regenerative Medicine, Guy's Hospital, King's College London, SE1 9RT, UK \\ ${ }^{2}$ Department of Haemato-Oncology, Rayne Institute, 123 Coldharbour Lane, London, SE5 9NU, UK \\ * Author for correspondence: curtisasante@gmail.com
}

\begin{abstract}
“The potential clinical applications of MSCs have generated much excitement within the regenerative medicine community but there is still a considerable lack of consensus within the field as to how MSCs exert their effects or even how to define them."
\end{abstract}

First draft submitted: 11 July 2017; Accepted for publication: 5 September 2017; Published online: 15 December 2017

Keywords: cell therapy $\bullet$ clinical translation $\bullet$ clinical trial $\bullet$ mesenchymal stromal cells $\bullet$ patient stratification $\bullet$ UK Regenerative Medicine Platform (UKRMP)

Mesenchymal stromal cells (MSCs) were first isolated from bone marrow but they can also be derived from other sources such as adipose tissue and umbilical cord. MSCs are non-hematopoietic cells that have been shown to generate some mesodermal cell types such as cartilage, bone and adipose cells in vitro and they are known to play important roles in hematopoeisis [1]. There is extensive literature on the immunosuppressive effects of MSCs [2] and consequently, these properties are currently being utilized in various cell therapy clinical trials for many indications. The potential clinical applications of MSCs have generated much excitement within the regenerative medicine community but there is still a considerable lack of consensus within the field as to how MSCs exert their effects or even how to define them. Therefore, coordinated multi-institutional and multinational efforts are needed to standardize approaches both at the bench and in the clinic.

This commentary summarizes proceedings from a workshop at the Centre of Stem Cells and Regenerative Medicine, Kings College London on 29-30 November 2016, to discuss and assess the current landscape of MSC research taking place across the UK and Europe. The workshop focused on current research in the field as well as the factors that both facilitate and impede the translation of preclinical results into effective treatment in the clinic. The workshop was hosted by both the Immunomodulation and Safety Hubs of the UK Regenerative Medicine Platform (UKRMP) and was attended by more than 50 international experts in cellular therapies. This commentary encapsulates the discussions that took place on the second day of the workshop, which focused on three separate themes: patient stratification for new trials, harmonization of preclinical and clinical studies at an international level, and expediting clinical trials. The following scientific experts were present: Andrew Hope (Biomedical Research Centre at Guy's and St Thomas' NHS Foundation Trust and King's College London, UK); Patricia Murray and Bettina Wilm (University of Liverpool and UKRMP, UK); Brigitte Wieles, Marlies Reinders and Koen Schepers (Leiden University, UK); Francesco Dazzi and Christina Trento (King's College London and UKRMP, UK); Ian McKay (Department of Health, UK); John Girdlestone (NHS Blood and Transplant, UK), and Tammy Kalber (University College London and UKRMP, UK).

\section{Patient stratification for new trials}

MSCs are being used in many clinical trials, the number of which is rapidly increasing. Furthermore, in the UK, cell therapies such as MSC therapies, which are intended for the clinic can be given a 'specials' license whereby the therapy is specifically manufactured or imported under the order of a doctor, dentist or prescriber for the treatment of individual patients. However, even though there are now numerous animal studies showing significant efficacy of MSCs in various diseases, including conditions that affect the heart, kidney, eye and spinal cord, results from 
clinical trials have been less convincing. A key problem is that the therapeutic mechanisms of action are ill-defined, making it very difficult to understand differences in efficacy between animal models and human patients as well as who should be treated with these therapies and how.

A clinical trial traditionally involves one drug treating one condition but in the MSC field, there is heterogeneity in the treatment approach and heterogeneity in the indications that are being treated. Although designating MSCs as 'specials' to be used on patients facilitates the availability of this therapy within a clinical trial setting, a large number of patients with the same indication will need to be treated in order to determine whether the treatment will actually be effective for everyone with that indication. Furthermore, in the UK, unless there is an NHS-wide approach with the relevant financial and infrastructure support to use MSCs under 'specials' administration, many hospitals may struggle to adopt this approach. As with many clinical trials, national multi-institutional and even multinational coordinated trials are needed although the latter will require negotiation of different regulatory practices and legislation that is adopted in different countries.

Patient engagement at an early stage is crucial as it informs patients of the implications of the clinical trials and reduces patient drop-out rates. For example, if a patient is enrolled in a randomized open label study, there is a real risk that the patient will drop out once they find out that they have been assigned to a control group which is not perceived by the patient to be of any benefit. It is important that the clinical trial team clearly explain the trial design at the beginning of the recruitment process as well as the aims, potential benefits and drawbacks. It is equally important that clinical trials are designed to remove investigator bias as much as possible by ensuring that where possible, studies are blinded and/or randomized.

\section{Harmonization of clinical \& preclinical studies at an international level}

The cost of clinical research is constantly rising due to the necessity to handle larger and larger data sets. This is partly driven by the demand for monitoring an increasing number of parameters with more stringent thresholds, which help to determine treatment efficacy. Yet, within the MSC field, there is a lack of readily available raw data from most MSC clinical trials from which to build upon when formulating new and improved clinical trials. One potential way to gather this data - at least in the UK - would be to tap into the English cancer registries, which gather patient-identifiable data to support cancer epidemiology, public health, service monitoring and research. These data are collected under legal permissions granted to Public Health England that were initially included under Section 60 of the Health and Social Care Act 2001 and more recently Section 251 of the NHS Act 2006.

Although no such registries exist specifically for MSC clinical trials, registries like the English cancer registries may be useful in those clinical trials where cancer is an indication that is being treated. Furthermore, MSC researchers should lobby government bodies that compile the registries to roll out similar systems that house data from MSC clinical trials. This would require a collaborative effort from all MSC research centers at an international level and an MSC consortium would be the best way to achieve this. This is an extremely important aspect for the success of future MSC clinical trials given that the ability to capture the differences in treatment regimens of different patients with different indications is required to understand and even predict how drugs work on different subgroups of people. The proposed MSC consortium would then determine the appropriate outputs and end points that will provide the data needed.

MSCs come from many different sources and are expanded in different ways. Christina Trento and Francesco Dazzi from King's College London and part of UKRMP are already carrying out detailed surveys with centers that are members of the European Society for Blood and Marrow Transplantation to determine their source of MSCs and how they expand, maintain and administer their MSCs. The data from these surveys will be hugely important in standardizing MSC preclinical and clinical research.

It is also worth considering whether there should be a facility that provides the required cells for all clinical trials at a national level. This would require a huge effort in terms of scale-up approaches and coordination between multiple facilities. MSC production and delivery approaches would need to be refined so that they are appropriate for different indications. While the cost of GMP scale-up would be substantial and would need to be accounted for in early planning stages, the benefit of standardization and scale-up cell preparation would be enormous for the comparability of clinical trial outcomes.

\section{Expediting clinical trials}

A major stumbling block of many clinical trial proposals is securing the funding to cover their considerable costs. National health services will always aim to treat people as economically and effectively as possible. Collaborative 
efforts with biotech companies at an early stage could be useful in these instances. For example, CliniMACS Prodigy ${ }^{\circledR}$ (Miltenyi Biotec, Bergisch Gladbach, Germany), is an integrated cell processing device, which can handle all current technical requirements for manufacturing cellular products by automation of the complete process in a functionally closed environment. This approach leads to automated, standardized cell manufacturing processes that can be carried out in a Grade D cleanroom (because the system is closed) rather than a Grade A cleanroom, which is much more expensive to operate in the long term.

To use MSCs in clinical trials within the NHS, appraisal of the study would be required by NICE. For NICE, quality of life is a crucially important way to measure efficacy and value of treatment. Assessment of the quality of life should be included throughout the duration of all MSC clinical trials by using simple questionnaires because it is very difficult to assess retrospectively.

Organizations like NHS Blood and Transplant (NHSBT) are well-placed to take over the burden of delivering cell therapy products under 'specials' status to the clinic. NHSBT already produces tissue derivatives, which are being produced clinically as advanced therapy medicinal products and collaboration with an MSC consortium could further facilitate the MSC therapies' route to the clinic.

\section{Conclusion}

Much progress has been made with MSC research from both preclinical and clinical studies but more can be done. The following action points were recommended to facilitate the translation of MSC research into clinical practice:

- Standardize expression profile assays for MSC classification

- Elucidate specific MSC mechanisms of action

- Standardize MSC manufacturing approaches

- Coordinate collaborative efforts with existing registries, for example, English cancer registries

- Coordinate collaborative efforts with organizations that routinely deliver therapies to the clinic, for example, NHSBT

- Increase collaborative efforts with biotechnology and pharmaceutical companies that have expertise in scale-up approaches

- Accurately assess cost of trials before applying for funding

- Form international MSC preclinical and clinical research consortiums

Financial \& competing interests disclosure

The workshop was funded by a grant from the UK Regenerative Medicine Platform (UKRMP). The authors have no other relevant affiliations or financial involvement with any organization or entity with a financial interest in or financial conflict with the subject matter or materials discussed in the manuscript apart from those disclosed.

No writing assistance was utilized in the production of this manuscript.

Open access

This work is licensed under the Attribution-NonCommercial-NoDerivatives 4.0 Unported License. To view a copy of this license, visit http://creativecommons.org/licenses/by-nc-nd/4.0/

\section{References}

1 Dazzi F, Ramasamy R, Glennie S, Jones S, Roberts I. The role of mesenchymal stem cells in haemopoiesis. Blood Rev. 20(3), 161-171 (2005).

2 Yarygin KN, Lupatov AY, Sukhikh GT. Modulation of immune responses by mesenchymal stromal cells. Bull. Exp. Biol. Med. 161(4), 561-565 (2016). 